\title{
The minimal entropy measure and an Esscher transform in an incomplete market model
}

\author{
Michael Monoyios \\ Mathematical Institute, University of Oxford, 24-29 St Giles', Oxford OX1 3LB, UK
}

Received 9 January 2006; accepted 17 January 2007

Available online 11 February 2007

\begin{abstract}
We consider an incomplete market model with one traded stock and two correlated Brownian motions $W$, $\widetilde{W}$. The Brownian motion $W$ drives the stock price, whose volatility and Sharpe ratio are adapted to the filtration $\widetilde{\mathbb{F}}:=\left(\widetilde{\mathscr{F}}_{t}\right)_{0 \leqslant t \leqslant T}$ generated by $\widetilde{W}$. We show that the projections of the minimal entropy and minimal martingale measures onto $\widetilde{\mathscr{F}}_{T}$ are related by an Esscher transform involving the correlation between $W, \widetilde{W}$, and the mean-variance trade-off process. The result leads to a new formula for the marginal exponential utility-based price of an $\widetilde{\mathscr{F}}_{T}$-measurable European claim. (C) 2007 Elsevier B.V. All rights reserved.
\end{abstract}

Keywords: Minimal entropy measure; Minimal martingale measure; Esscher transform

\section{Introduction}

In this paper we consider a two-factor continuous-time incomplete market model without asset price jumps. The model comprises two correlated Brownian motions $W, \widetilde{W}$, with fixed correlation $\rho \in[-1,1]$. The Brownian motion $W$ drives a stock price process $S$, and the parameters of the stochastic differential equation for $S$ are progressively measurable with respect to the filtration $\widetilde{\mathbb{F}}:=(\widetilde{\mathscr{F}})_{0 \leqslant t \leqslant T}$ generated by $\widetilde{W}$. Many stochastic volatility models fit into this framework, though we do not rely on a Markovian diffusion structure. This class of models has been studied by Tehranchi (2004) in particular.

The main result is that the projections of the minimal entropy martingale measure $Q^{\mathrm{E}}$ and the minimal martingale measure $Q^{\mathrm{M}}$ onto the sigma-field $\widetilde{\mathscr{F}}_{T}$ are related by an Esscher transform involving the correlation $\rho$ and the mean-variance trade-off process at time $T, K_{T}:=\int_{0}^{T} \lambda_{t}^{2} \mathrm{~d} t$, where $\lambda$ is the Sharpe ratio of the stock. Some related characterisations of martingale measures in such models have appeared in the literature, notably in Hobson (2004) and Rheinländer (2005), but the succinct result we report here has not been previously observed.

Our result can be seen as recasting the representation equations in Hobson (2004) and Rheinländer (2005), and also as providing a striking consequence of the well-known distortion power technique (Tehranchi, 2004; Zariphopoulou, 2001) for solving utility maximisation problems in such incomplete models. Finally, our result

E-mail address: monoyios@maths.ox.ac.uk. 
leads to a new formula for the marginal utility-based price (Davis, 1997) of an $\widetilde{\mathscr{F}}_{T}$-measurable European claim in this incomplete market.

In exponential indifference pricing, it is well known that the marginal price of the claim (Davis, 1997) is the expectation of the payoff (suitably discounted) under the minimal entropy measure. With logarithmic utility the corresponding measure is the minimal martingale measure $Q^{\mathrm{M}}$, and this measure is also prominent in quadratic hedging approaches. The Esscher transform between these measures can be viewed as quantifying the extra premium charged by an agent with exponential utility compared with an agent who has a logarithmic or quadratic criterion.

In the distortion power solution to utility maximisation problems, a value function $u(x)=E U\left(X_{T}^{*}\right)$, in which $U$ is the utility function, $X_{T}^{*}$ is optimal terminal wealth and $x$ is initial wealth, is obtained in the form

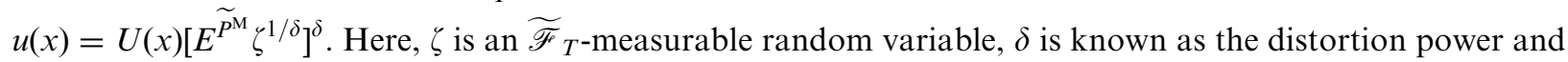
depends on the correlation $\rho$ (and, with power utility, on the risk aversion parameter), and $\widetilde{P}^{\mathrm{M}}$ is a measure which, with exponential utility, is the projection of $Q^{\mathrm{M}}$ onto $\widetilde{\mathscr{F}}_{T}$. Our results stem from translating this representation into a representation for the solution of the dual to the primal utility maximisation problem, and combining this with the representation equation in Hobson (2004) and Rheinländer (2005).

\section{The market model and main theorem}

Let $(\Omega, \mathscr{F}, P)$ be a probability space supporting two correlated Brownian motions $W:=\left(W_{t}\right)_{0 \leqslant t \leqslant T}$ and $\widetilde{W}:=\left(\widetilde{W}_{t}\right)_{0 \leqslant t \leqslant T}$ with fixed correlation $\rho \in[-1,1]$. Let $\mathbb{F}:=\left(\mathscr{F}_{t}\right)_{0 \leqslant t \leqslant T}$ be the completion of the filtration generated by the pair $(W, \widetilde{W})$, and denote by $\widetilde{\mathbb{F}}:=(\widetilde{\mathscr{F}})_{0 \leqslant t \leqslant T}$ the completion of the filtration generated by $\widetilde{W}$.

A stock price $S:=\left(S_{t}\right)_{0 \leqslant t \leqslant T}$ is driven by the Brownian motion $W$, and is described by

$$
\mathrm{d} S_{t}=\sigma_{t} S_{t}\left(\lambda_{t} \mathrm{~d} t+\mathrm{d} W_{t}\right)
$$

We work in a world with zero interest rates, so that $S$ represents a discounted price. The volatility $\left(\sigma_{t}\right)_{0 \leqslant t \leqslant T}$ and Sharpe ratio (or market price of risk) $\left(\lambda_{t}\right)_{0 \leqslant t \leqslant T}$ are progressively measurable processes with the volatility bounded away from zero (further integrability conditions are given later). Aside from constant correlation, the crucial assumption we make is the following.

Property 1. The volatility $\sigma_{t}$ and Sharpe ratio $\lambda_{t}$ are $\widetilde{\mathscr{F}}_{t}$-measurable for all $t \in[0, T]$.

In particular, this implies that the increasing process $K_{t}:=\int_{0}^{t} \lambda_{u}^{2} \mathrm{~d} u, 0 \leqslant t \leqslant T$, sometimes called the meanvariance trade-off process, is $\widetilde{\mathbb{F}}$-adapted.

Remark 1. The above assumption is restrictive, but encompasses stochastic volatility models (Fouque et al., 2000; Hobson, 2004), without requiring a Markovian structure. One could associate the volatility with another Itô process $Y$, writing $\sigma_{t}=f\left(t, Y_{t}\right)$ for some function $f$, with $Y$ following a process of the form:

$$
\mathrm{d} Y_{t}=a_{t} \mathrm{~d} t+b_{t} \mathrm{~d} \tilde{W}_{t},
$$

and yet more structure could be added by assuming the processes $\lambda, a, b$ are also functions of $Y$, making the model Markovian. Our analysis is valid without such assumptions.

Consider the class $\mathscr{M}$ of equivalent local martingale measures $Q \sim P$ on $\mathscr{F}_{T}$ with density processes given by

$$
\left.Z_{t} \equiv \frac{\mathrm{d} Q}{\mathrm{~d} P}\right|_{\mathscr{F}_{t}}=\mathscr{E}\left(-\lambda \cdot W-\psi \cdot W^{\perp}\right)_{t}, \quad 0 \leqslant t \leqslant T,
$$

where $\mathscr{E}$ is the Doléans exponential and $W^{\perp}$ is a Brownian motion independent of $W$. Here $\psi$ is an $\mathbb{F}$-adapted process, and we assume $\lambda, \psi$ are such that the Novikov condition $E \exp \left(\frac{1}{2}\left(K_{T}+\int_{0}^{T} \psi_{t}^{2} \mathrm{~d} t\right)\right)<\infty$ is satisfied, so that $\left(Z_{t}\right)_{0 \leqslant t \leqslant T}$ is a $(P, \mathbb{F})$-martingale, and measures $Q \in \mathscr{M}$ are equivalent to $P$.

The minimal martingale measure $Q^{\mathrm{M}}$ of Föllmer and Schweizer (1991) corresponds to $\psi_{t}=0,0 \leqslant t \leqslant T$. For models with continuous price trajectories, $Q^{\mathrm{M}}$ minimises the reverse relative entropy $H(P, Q):=$ $E[-\log (\mathrm{d} Q / \mathrm{d} P)]$ over all $Q \in \mathscr{M}$ (Schweizer, 1999), though the original appearance of this measure in 
finance was in a quadratic hedging context. Under $Q^{\mathrm{M}}$ the traded asset price becomes a local martingale, but the drift of any Brownian motion orthogonal to $W$ is left unchanged. This corresponds to leaving unhedgeable risk unpriced.

The minimal entropy martingale measure $Q^{\mathrm{E}}$ is defined by

$$
Q^{\mathrm{E}}:=\arg \min _{Q \in \mathscr{M}} H(Q, P),
$$

where $H(Q, P)$ is the relative entropy of $Q \in \mathscr{M}$ with respect to $P$ :

$$
H(Q, P):= \begin{cases}E\left(\frac{\mathrm{d} Q}{\mathrm{~d} P} \log \frac{\mathrm{d} Q}{\mathrm{~d} P}\right) & \text { if } Q \ll P \text { on } \mathscr{F}_{T}, \\ +\infty & \text { otherwise. }\end{cases}
$$

Let $\widetilde{Q}^{\mathrm{E}}, \widetilde{Q}^{\mathrm{M}}$ denote the projections of $Q^{\mathrm{E}}, Q^{\mathrm{M}}$ onto $\widetilde{\mathscr{F}}_{T}$. A simple calculation establishes that

$$
\frac{\mathrm{d} \widetilde{Q}^{\mathrm{M}}}{\mathrm{d} P}=\mathscr{E}(-\rho \lambda \cdot \widetilde{W})_{T}
$$

Similarly, suppose that $Q^{\mathrm{E}}$ is given by

$$
\frac{\mathrm{d} Q^{\mathrm{E}}}{\mathrm{d} P}=\mathscr{E}\left(-\lambda \cdot W-\xi \cdot W^{\perp}\right)_{T},
$$

for some choice $\psi=\xi$ of the integrand in (1). Then $\widetilde{Q}^{\mathrm{E}}$ is given by

$$
\frac{\mathrm{d} \widetilde{Q}^{\mathrm{E}}}{\mathrm{d} P}=\mathscr{E}[-(\rho \lambda+\bar{\rho} \xi) \cdot \widetilde{W}]_{T}
$$

where $\bar{\rho}=\sqrt{1-\rho^{2}}$.

The following theorem is the main result of the paper.

Theorem 1. The measures $Q^{\mathrm{M}}, Q^{\mathrm{E}}$, projected onto the sigma-field $\widetilde{\mathscr{F}}_{T}$, are related by the Esscher transform

$$
\frac{\mathrm{d} \widetilde{Q}^{\mathrm{E}}}{\mathrm{d} \widetilde{Q}^{\mathrm{M}}}=\frac{\exp \left(\theta K_{T}\right)}{E^{Q^{\mathrm{M}}} \exp \left(\theta K_{T}\right)},
$$

where $\theta=-\frac{1}{2}\left(1-\rho^{2}\right)$ and $K_{T}=\int_{0}^{T} \lambda_{t}^{2} \mathrm{~d} t$ is the mean-variance trade-off at $T$.

Esscher transforms have a long history in actuarial pricing, and have been used by some authors to define a possible pricing measure in incomplete markets (Bühlmann et al., 1996; Gerber and Shiu, 1994). In this context the Esscher transform is quantifying the "economic premium" (Bühlmann, 1980) associated with pricing under the minimal entropy measure $Q^{\mathrm{E}}$ (and hence with exponential hedging) over and above pricing with the minimal measure $Q^{\mathrm{M}}$ (and essentially leaving unhedgeable risk unpriced).

\section{Proofs}

The proof of Theorem 1 rests on fusing two results. The first is a representation equation of Hobson (2004) for the so-called $q$-optimal measure $(q \in \mathbb{R})$ in stochastic volatility models. For $q=1$, the $q$-optimal measure is the minimal entropy measure, and Hobson's result reduces to a representation equation of Rheinländer (2005). The second result is the well-known distortion solution (Tehranchi, 2004; Zariphopoulou, 2001) for utility maximisation problems in two-factor incomplete markets, suitably translated into a representation for the optimal measure of the dual to the primal utility maximisation problem.

For $q=1$, the Hobson representation equation is as follows.

Proposition 1 (Hobson, 2004; Rheinländer, 2005). Suppose there are previsible processes $\eta$ and $\xi$ and a finite constant $c$ such that

$$
\frac{1}{2} K_{T}=M_{T}+L_{T}+\frac{1}{2}[L]_{T}+c,
$$


where

$$
M_{t}:=\int_{0}^{t} \eta_{u}\left(\mathrm{~d} W_{u}+\lambda_{u} \mathrm{~d} u\right), \quad L_{t}:=\int_{0}^{t} \xi_{u} \mathrm{~d} W_{u}^{\perp} .
$$

Then, provided $\int_{0}^{T}\left(\eta_{t}-\lambda_{t}\right)^{2} \mathrm{~d} t$ has an exponential moment under $P$, the minimal entropy measure $Q^{\mathrm{E}}$ is given by

$$
\frac{\mathrm{d} Q^{\mathrm{E}}}{\mathrm{d} P}=\mathscr{E}\left(-\lambda \cdot W-\xi \cdot W^{\perp}\right)_{T},
$$

and the constant $c$ is given by

$$
c=H\left(Q^{\mathrm{E}}, P\right) \equiv E\left[\frac{\mathrm{d} Q^{\mathrm{E}}}{\mathrm{d} P} \log \frac{\mathrm{d} Q^{\mathrm{E}}}{\mathrm{d} P}\right] .
$$

The salient point concerning the above proposition is that, provided a solution to (4) is found, in the form of the processes $\eta, \xi$ and the constant $c$, then the minimal entropy measure is identified via (5), provided certain integrability conditions are satisfied.

The second ingredient we need to prove Theorem 1 is the distortion power solution (Tehranchi, 2004) for utility maximisation problems in models such as ours. The consequences of this solution for the dual problem will be exploited to obtain an alternative expression for the constant $c$ in (4). Combining all these results will lead to our final theorem.

The primal utility maximisation problem is as follows. Given an initial endowment $x$, an investor who forms a self-financing portfolio involving $S$ will generate wealth process $X:=\left(X_{t}\right)_{0 \leqslant t \leqslant T}$ given by

$$
X_{t}=x+\int_{0}^{t} \sigma_{u} \pi_{u} \lambda_{u} \mathrm{~d} u+\int_{0}^{t} \sigma_{u} \pi_{u} \mathrm{~d} W_{u},
$$

where $\pi:=\left(\pi_{t}\right)_{0 \leqslant t \leqslant T}$ is the wealth held in the stock and represents the agent's trading strategy. We write $X \equiv X^{\pi}$ if we need to emphasise dependence on $\pi$ at any point. A trading strategy is an adapted process $\pi$ satisfying $\int_{0}^{t} \sigma_{u}^{2} \pi_{u}^{2} \mathrm{~d} u<\infty$ for all $t \in[0, T]$, so that the stochastic integral in (7) is well defined.

With an exponential utility function $U(x)=-\exp (-\gamma x), \gamma>0, x \in \mathbb{R}$, the objective is to maximise expected utility of wealth at time $T$, over some class $\mathscr{A}$ of admissible trading strategies. The value function is

$$
u(x):=\sup _{\pi \in \mathscr{A}} E U\left(X_{T}^{\pi}\right) .
$$

A minimal assumption on the set $\mathscr{A}$ of admissible strategies is such that the expected utility $E U\left(X_{T}^{\pi}\right)$ is well defined for all $\pi \in \mathscr{A}$. However, to exclude pathologies such as "doubling strategies" one usually imposes further integrability on the set of admissible strategies. We follow Tehranchi (2004), and define $\mathscr{A}$ as the set of strategies $\pi$ satisfying $E \sup _{t \in[0, T]} \exp \left(-\gamma^{\prime} X_{t}^{\pi}\right)<\infty$ for some $\gamma^{\prime}>\gamma$. There are other possible characterisations of admissibility in exponential utility maximisation problems, and these are discussed in depth in Delbaen et al. (2002) and Schachermayer (2001), to which the interested reader is referred.

We then have the following proposition, due to Tehranchi (2004), which extends results of Zariphopoulou (2001) to a non-Markovian scenario.

Proposition 2 (Tehranchi, 2004). The value function in (8) is given by

$$
u(x)=U(x)\left[E^{\widetilde{Q}^{\mathrm{M}}} \exp \left(\theta K_{T}\right)\right]^{1 /\left(1-\rho^{2}\right)},
$$

where $\widetilde{Q}^{\mathrm{M}}$ is the projection of the minimal martingale measure onto $\widetilde{\mathscr{F}}_{T}$, given by (2), and $\theta=-\frac{1}{2}\left(1-\rho^{2}\right)$.

We shall also use the convex dual to this problem. Let $V: \mathbb{R}^{+} \rightarrow \mathbb{R}$ denote the convex conjugate of the utility function $U$ :

$$
V(y):=\sup _{x \in \mathbb{R}}[U(x)-x y], \quad y>0,
$$


satisfying the bidual relation

$$
U(x)=\inf _{y \in \mathbb{R}^{+}}[V(y)+x y], \quad x \in \mathbb{R} .
$$

The dual problem is to minimise the expectation of $V(y \mathrm{~d} Q / \mathrm{d} P)$ over local martingale measures $Q \in \mathscr{M}$, and its value function is

$$
v(y):=\inf _{Q \in \mathscr{M}} E V\left(y \frac{\mathrm{d} Q}{\mathrm{~d} P}\right) .
$$

We need a lemma relating the constant $c=H\left(Q^{\mathrm{E}}, P\right)$ in (6) to the expectation in (9).

Lemma 1. The constant $c=H\left(Q^{\mathrm{E}}, P\right)$ in (6) is given by

$$
c=H\left(Q^{\mathrm{E}}, P\right)=-\frac{1}{1-\rho^{2}} \log E^{\widetilde{Q}^{\mathrm{M}}} \exp \left(\theta K_{T}\right)
$$

where $\theta=-\frac{1}{2}\left(1-\rho^{2}\right)$.

Proof. For $U(x)=-\exp (-\gamma x)$ we have, from (10), that $V(y)=(y / \gamma)(\log (y / \gamma)-1)$, and so (11) gives

$$
v(y)=V(y)+\frac{y}{\gamma} H\left(Q^{\mathrm{E}}, P\right) .
$$

It is well known (for example Delbaen et al., 2002; Schachermayer, 2001) that the value functions $u(x)$ and $v(y)$ are conjugate:

$$
v(y)=\sup _{x \in \mathbb{R}}[u(x)-x y], \quad u(x)=\inf _{y \in \mathbb{R}^{+}}[v(y)+x y] .
$$

Using this with (12) connects the primal value function with the minimal entropy:

$$
u(x)=-\exp \left[-\gamma x-H\left(Q^{\mathrm{E}}, P\right)\right] .
$$

Comparing this with Proposition 2 gives

$$
\exp \left[-H\left(Q^{\mathrm{E}}, P\right)\right]=\left[E^{\widetilde{Q}^{\mathrm{M}}} \exp \left(\theta K_{T}\right)\right]^{1 /\left(1-\rho^{2}\right)},
$$

from which the result follows.

Proof of Theorem 1. Since the Brownian motions $W, \widetilde{W}$ have correlation $\rho$, write $\widetilde{W}=\rho W+\bar{\rho} W^{\perp}$, where $\bar{\rho}=\sqrt{1-\rho^{2}}$ and $W^{\perp}$ is orthogonal to $W$. Define also a Brownian motion $\widetilde{W}^{\perp}$ orthogonal to $\widetilde{W}$ by

$$
\widetilde{W}^{\perp}:=\bar{\rho} W-\rho W^{\perp} .
$$

We have, using (2) and (3),

$$
\begin{aligned}
\frac{\mathrm{d} \widetilde{Q}^{\mathrm{E}}}{\mathrm{d} \widetilde{Q}^{\mathrm{M}}} & =\frac{\mathrm{d} \widetilde{Q}^{\mathrm{E}}}{\mathrm{d} P} / \frac{\mathrm{d} \widetilde{Q}^{\mathrm{M}}}{\mathrm{d} P} \\
& =\frac{\mathscr{E}[-(\rho \lambda+\bar{\rho} \xi) \cdot \widetilde{W}]_{T}}{\mathscr{E}(-\rho \lambda \cdot \widetilde{W})_{T}} \\
& =\mathscr{E}(-\bar{\rho} \xi \cdot \widetilde{W})_{T} \exp \left(-\rho \bar{\rho} \int_{0}^{T} \lambda_{t} \xi_{t} \mathrm{~d} t\right) .
\end{aligned}
$$

Note that since the left-hand side is $\widetilde{\mathscr{F}} T$-measurable, this implies that the process $\xi$ identifying the minimal entropy measure in our model is $\widetilde{\mathbb{F}}$-adapted. We shall use this shortly. 
Now use the fundamental representation equation (4), which we re-write in terms of the Brownian motions $\widetilde{W}, \widetilde{W}^{\perp}$, and use Lemma 1 to substitute for the constant $c$, giving (4) in the form

$$
\begin{aligned}
\frac{1}{2} K_{T}= & \int_{0}^{T}\left(\rho \eta_{t}+\bar{\rho} \xi_{t}\right) \mathrm{d} \widetilde{W}_{t}+\int_{0}^{T}\left(\bar{\rho} \eta_{t}-\rho \xi_{t}\right) \mathrm{d} \widetilde{W}_{t}^{\perp}+\int_{0}^{T}\left(\eta_{t} \lambda_{t}+\frac{1}{2} \xi_{t}^{2}\right) \mathrm{d} t \\
& -\frac{1}{1-\rho^{2}} \log E^{\widetilde{Q}^{\mathrm{M}}} \exp \left(\theta K_{T}\right) .
\end{aligned}
$$

Now, the left-hand side of (14) is $\widetilde{\mathscr{F}} T$-measurable, and we know that $\xi$ is also $\widetilde{\mathbb{F}}$-adapted. Therefore, to find a process $\eta$ that satisfies (14) we need to eliminate the stochastic integral with respect to $\widetilde{W}^{\perp}$, so that $\eta$ must satisfy

$$
\bar{\rho} \eta_{t}-\rho \xi_{t}=0, \quad 0 \leqslant t \leqslant T .
$$

With this choice of $\eta$, (14) becomes, on multiplying by $\bar{\rho}^{2}=1-\rho^{2}$ and re-arranging terms,

$$
-\bar{\rho}(\xi \cdot \widetilde{W})_{T}-\frac{1}{2} \bar{\rho}^{2} \int_{0}^{T} \xi_{t}^{2} \mathrm{~d} t-\rho \bar{\rho} \int_{0}^{T} \lambda_{t} \xi_{t} \mathrm{~d} t=-\frac{1}{2} \bar{\rho}^{2} K_{T}-\log E^{\widetilde{Q}^{\mathrm{M}}} \exp \left(\theta K_{T}\right),
$$

or, equivalently,

$$
\mathscr{E}(-\bar{\rho} \xi \cdot \widetilde{W})_{T} \exp \left(-\rho \bar{\rho} \int_{0}^{T} \lambda_{t} \xi_{t} \mathrm{~d} t\right)=\frac{\exp \left(-\frac{1}{2} \bar{\rho}^{2} K_{T}\right)}{E^{Q^{\mathrm{M}}} \exp \left(\theta K_{T}\right)},
$$

and the result follows from (13).

\section{A new formula for the marginal price of a claim}

A corollary of Theorem 1 is the following representation, in incomplete models of the class studied here, for the marginal exponential-utility-based price (Davis, 1997) of a European claim paying an $\mathscr{\mathscr { F }}_{T}$-measurable random variable $\widetilde{G}$ at time $T$.

Corollary 1. With exponential utility, the marginal price of a European $\widetilde{\mathscr{F}}_{T}$-measurable claim $\widetilde{G}$ has the representation

$$
\tilde{p}=\frac{E^{\widetilde{Q^{\mathrm{M}}}}\left[\exp \left(\theta K_{T}\right) \widetilde{G}\right]}{E^{\widetilde{Q^{\mathrm{M}}}}\left[\exp \left(\theta K_{T}\right)\right]} .
$$

Proof. The marginal utility-based price of Davis (1997) has, with exponential utility, a representation as the $Q^{\mathrm{E}}$-expectation (suitably discounted) of the payoff (see Becherer, 2003 for example). When the payoff is $\widetilde{\mathscr{F}}_{T^{-}}$ measurable this expectation reduces to one under $\widetilde{Q}^{\mathrm{E}}$, and the result is immediate on writing $\widetilde{p}=E^{\mathrm{Q}} \widetilde{G}$ and using Theorem 1 to express the price as a $\widetilde{Q}^{\mathrm{M}}$-expectation.

\section{References}

Becherer, D., 2003. Rational hedging and valuation of integrated risks under constant absolute risk aversion. Insurance Math. Econom. $33,1-28$.

Bühlmann, H., 1980. An economic premium principle. ASTIN Bull. 11, 52-60.

Bühlmann, H., Delbaen, F., Embrechts, P., Shiryaev, A.S., 1996. No-arbitrage, change of measure and conditional Esscher transforms. CWI Quarterly 9, 291-317.

Davis, M.H.A., 1997. Option pricing in incomplete markets. In: Dempster, M.A.H., Pliska, S.R. (Eds.), Mathematics of Derivative Securities. Cambridge University Press, Cambridge, pp. 216-226.

Delbaen, F., Grandits, P., Rheinländer, T., Samperi, D., Schweizer, M., Stricker, C., 2002. Exponential hedging and entropic penalties. Math. Finance 12, 99-123.

Föllmer, H., Schweizer, M., 1991. Hedging of contingent claims under incomplete information. In: Davis, M.H.A., Elliott, R.J. (Eds.), Applied Stochastic Analysis, Stochastics Monographs, vol. 5. Gordon \& Breach, New York, pp. 389-414. 
Fouque, J.P., Papanicolaou, G., Sircar, R., 2000. Derivatives in Financial Markets with Stochastic Volatility. Cambridge University Press, Cambridge.

Gerber, H.U., Shiu, E.S.W., 1994. Option pricing by Esscher transforms. Trans. Soc. Actuaries 46, 99-191.

Hobson, D.G., 2004. Stochastic volatility models, correlation, and the $q$-optimal measure. Math. Finance 14, 537-556.

Rheinländer, T., 2005. An entropy approach to the Stein-Stein model with correlation. Finance \& Stochastics 9, $399-413$.

Schachermayer, W., 2001. Optimal investment in incomplete markets when wealth may become negative. Ann. Appl. Probab. 11, 694-734.

Schweizer, M., 1999. A minimality property of the minimal martingale measure. Statist. Probab. Lett. 42, $27-31$.

Tehranchi, M., 2004. Explicit solutions of some utility maximization problems in incomplete markets. Stochastic Process. Appl. 114, 109-125.

Zariphopoulou, T., 2001. A solution approach to valuation with unhedgeable risks. Finance \& Stochastics 5, 61-82. 